\title{
A method to reduce DC-link voltage fluctuation of PMSM drive system with reduced DC-link capacitor
}

\author{
Ke Li, Yi Wang ${ }^{\mathrm{a}}$, Hong Wang and Danyang Bao \\ Power Electronics and Electrical Drives Research Center, Harbin Institute of Technology Shenzhen Graduate \\ School, Shenzhen, China
}

\begin{abstract}
The reduction of the dc-link capacitance in the motor drive makes it possible to improve the system power density and lower the system cost. However, this can lead to relatively high dc-link voltage fluctuation, especially the sixth harmonic component. To serve the purpose of enhancing the dc-link voltage quality, a parameter-insensitive control method was proposed in this paper. The proposed method shapes the sixth harmonic component of the dc-link current in phase with that of the inductor current by the HF power injection technology. Since this practice can reduce the difference between these two sixth harmonic components, the sixth harmonic component of the dc-link voltage can be reduced as well. The simulation results show that the proposed method not only improves the ripple characteristic of dc-link voltage, but also achieves the aim of PMSM speed regulation.
\end{abstract}

Keywords: DC-link capacitor; permanent magnet synchronous motor (PMSM); power injection; voltage fluctuation; voltage source inverter (VSI).

\section{Introduction}

Permanent magnet synchronous motors (PMSMs) have attractive advantages, such as high efficiency, high precision, high torque to inertia ratio, and high power density, gaining widespread acceptance in various applications [1-2]. In conventional motor drive, large electrolytic capacitors are usually installed to maintain constant dc-link voltage, which occupy considerable space and increase the system cost. Given these issues, a lot of efforts have been spent to reduce or minimize the dc-link capacitance.

In [3-4], the dc-link capacitance was reduced to a very small value, and both the motor speed regulation and the input power factor improvement were achieved by controlling the inverter output power. In [5], a new current injection circuit together with a current control method were proposed for the motor drive system with small dc-link capacitor, and such practice makes it possible to meet the standard of input harmonic current for motor drive. It is worth noting that the significant reduction of dc-link capacitance can result in the instability problem, a dc-link voltage stability method based on active damping was presented in [6] accordingly, increasing the system stability. Actually, under the precondition of stable system, the dc-link voltage fluctuation caused by the reduction of dc-link capacitance is still relatively high, which is worth investigating as well. Therefore, Bon-Gwan Gu proposed a control method to limit the dc-link voltage variation in a converter-inverter system with

\footnotetext{
a Corresponding author : wangyi_hitsz@163.com, like_hitsz@163.com
} 
reduced dc-link capacitor [7], which was based on the directly control of dc-link capacitor current. Since this method requires a controllable front-end rectifier, it cannot be applied to the diode-rectifierbased system. Unlike the practice in [7], the dc-link voltage fluctuation was suppressed by only regulating the motor reactively power in [8]. Such principle can be used for reference in the dioderectifier-based system. However, this method looks somewhat complex and depends on the knowledge of motor parameters.

This study focuses on the dc-link voltage fluctuation problem of the topology which consists of a three-phase diode rectifier, an $L C$ filter with reduced capacitor, and a voltage source inverter (VSI). Then, a novel control method, which is independent of motor parameters, is proposed. Using the proposed method, the difference between the sixth harmonic component of indictor current and that of the dc-link current is reduced. Also, the dc-link voltage fluctuation is reduced accordingly. Simulation results are provided to confirm the feasibility and effectiveness of the presented method.

\section{Drive system configuration and problem description}

\subsection{Drive system configuration}

The PMSM drive system with reduced dc-link capacitor is shown in Fig. 1, which is equipped with a six-pulse diode rectifier, an $L C$ filter with reduced capacitor, and a VSI. In such system, the combination of the VSI and the PMSM can be simply considered as an equivalent load of the frontend circuit $[6,9]$. In order to understand the characteristics of the equivalent load, the model of the PMSM should be established first. For simplicity, the PMSM is commonly modelled in the $d q$ reference frame, and the corresponding equations are given by

$$
\begin{gathered}
u_{d}=R i_{d}+L_{d} \frac{d i_{d}}{d t}-\omega_{e} L_{q} i_{q} \\
u_{q}=R i_{q}+L_{q} \frac{d i_{q}}{d t}+\omega_{e} L_{d} i_{d}+\omega_{e} \psi_{f} \\
p_{e}=\frac{3}{2}\left(u_{d} i_{d}+u_{q} i_{q}\right)
\end{gathered}
$$

Where $u_{d}$ and $u_{q}$ are the $d$ - and $q$-axes stator voltages, $i_{d}$ and $i_{q}$ are the $d$ - and $q$-axes stator currents, $R$ is the stator resistance, $L_{d}$ and $L_{q}$ are the $d$ - and $q$-axes stator inductances, $\omega_{e}$ is the electrical angular speed, $\psi_{f}$ is the permanent magnet flux linkage, and $p_{e}$ is the electric power of the PMSM, respectively. Under conventional field-oriented control, the steady-state stator currents ( $i_{d}$ and $i_{q}$ ) as well as the electrical angular speed can be regarded as constant. Then, it follows from (1) to (3) that the electric power of the PMSM, $p_{e}$, can also be regarded as constant. Further, neglecting the power loss of the VSI, the input power of the VSI and the electric power of the PMSM can be considered equivalent, and hence, the aforementioned equivalent load can be modelled as a constant power load [9]. In this case, the PMSM drive system in Fig. 1 using conventional field-oriented control can be simplified accordingly, as shown in Fig. 2. 


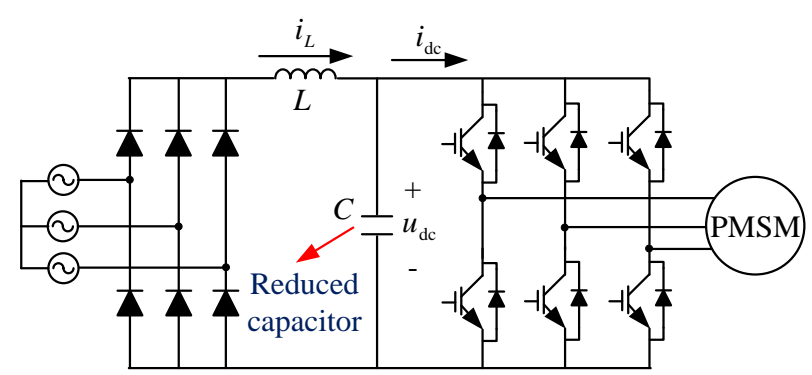

Figure 1. PMSM drive system with reduced dc-link capacitor.

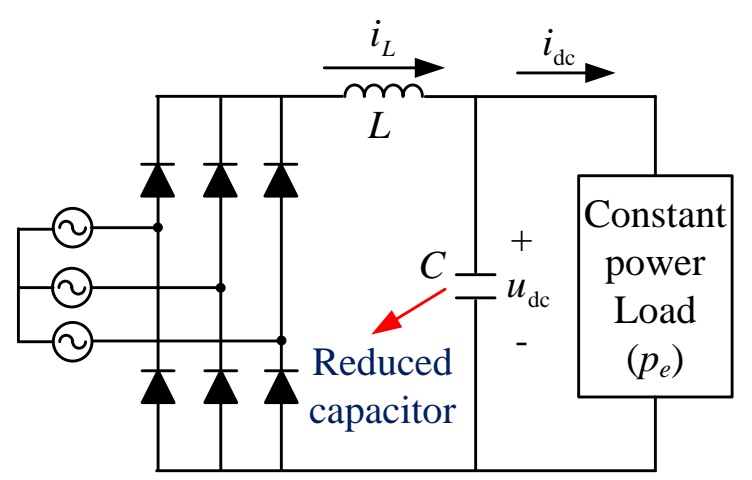

Figure 2. Simplified model for system with reduced dc-link capacitor under conventional field-oriented control.

\subsection{Problem description}

Typically, the diodes in the six-pulse rectifier are alternately turned on and off. Therefore, the dc-link voltage $u_{\mathrm{dc}}$ can suffer some harmonics, especially the harmonic with six times the grid frequency. In this paper, the dc-link capacitor is reduced, which can save the space and the cost of the drive system. However, it makes the cut-off frequency of the $L C$ filter higher than six times the grid frequency. In other words, provided that the dc-link capacitor is reduced, the sixth harmonic problem of the dc-link voltage will become more significant.

To clearly demonstrate the relationship between the dc-link voltage and the dc-link capacitance, Fig. 3 shows the dc-link voltages under the same constant power load $\left(p_{e}=2 \mathrm{~kW}\right)$ with different dc-link capacitances. It can be seen that the smaller the capacitance, the higher the voltage ripple is. This means that, the voltage fluctuation in the drive system with reduced dc-link capacitor is relatively high. Furthermore, since the voltage ripple is the main factor causing the temperature rise of the capacitor, it should be suppressed in the drive system with reduced dc-link capacitor.

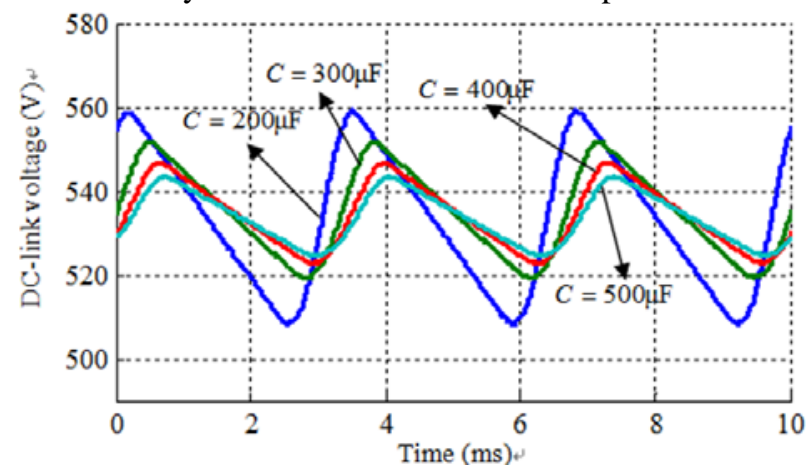

Figure 3. DC-link voltages with different dc-link capacitances. 


\section{Proposed control method for reducing voltage fluctuation}

\subsection{Principe of reducing voltage fluctuation}

In the PMSM derive system, the dc-link capacitor current $i_{\mathcal{C}}$ which lead to a dc-link voltage fluctuation, can be expressed by the inductor current $i_{L}$ and the dc-link current $i_{\mathrm{dc}}$, namely,

$$
i_{c}=C \frac{d u_{\mathrm{dc}}}{d t}=i_{L}-i_{\mathrm{dc}}
$$

It implies that to deal with the dc-link voltage fluctuation problem the difference between $i_{L}$ and $i_{\mathrm{dc}}$ should be reduced. Furthermore, because the dominant harmonic component of dc-link voltage is sixth harmonic component, the sixth harmonic current difference between $i_{L}$ and $i_{\mathrm{dc}}$ will be only concerned in this paper. Neglecting the power loss of the VSI, $p_{e}$ can also be described as

$$
p_{e}=u_{\mathrm{dc}} i_{\mathrm{dc}}
$$

Therefore, the sixth harmonic component of $i_{\mathrm{dc}}$ can be controlled by injecting high frequency (HF) components into $p_{e}$. In this case, similar to the simplification shown in Fig. 2, the PMSM and the VSI can also be modeled as an equivalent load whose power is $p_{e}$. However, due to the HF power injection, the motor electric power $p_{e}$ is no longer constant, and some HF components are involved in it. Then, the equivalent load can be considered as a combination of the constant power load and adjustable HF power load, as demonstrated in Fig. 4.

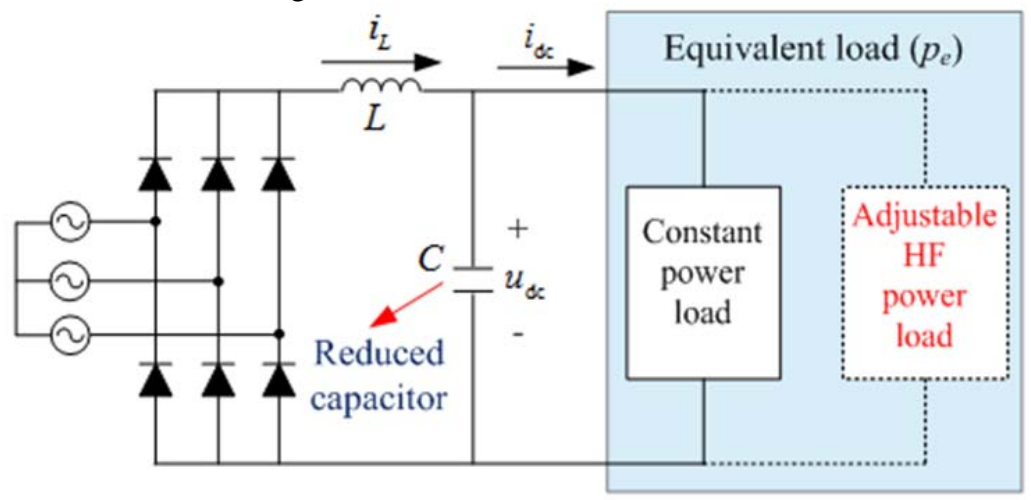

Figure 4. Simplified model for system with reduced dc-link capacitor under HF power injection.

Furthermore, it follows from (3) that the HF power injection for $p_{e}$ (i.e., the adjustable HF power load in Fig. 4) can be implemented by adjusting the $q$-axis stator current of PMSM. Hence, no additional circuit is required for this power injection.

In the studied drive system, the reduced dc-link capacitor still have relatively strong energy decoupling effect, the adjustment to sixth harmonic component of $i_{\mathrm{dc}}$ has little influence on that of $i_{L}$. In this case, the purpose of reducing the sixth harmonic current difference between $i_{L}$ and $i_{\mathrm{dc}}$ can be served by shaping the sixth harmonic component of $i_{\mathrm{dc}}$ in phase with that of $i_{L}$. Further, the sixth harmonic voltage will be reduced accordingly. As the shaping of the sixth harmonic component of $i_{\mathrm{dc}}$ depends on the HF components of motor electric power, the input power requirement of VSI to shape the sixth harmonic component of $i_{\mathrm{dc}}$ will be analyzed in the following sections.

\subsection{Input power requirement of VSI}

The dc-link current $i_{\mathrm{dc}}$ after shaping can be expressed as (6), which is composed of the DC component $i_{\mathrm{dc} 0}$ and the shaped sixth harmonic component $i_{d c 6}$. 


\section{AEST2016}

$$
i_{\mathrm{dc}}=i_{\mathrm{dc} 0}+i_{\mathrm{dc} 6}
$$

Moreover, as aforementioned analysis, $i_{\mathrm{dc} 6}$ should be shaped in phase with the sixth harmonic component of $i_{L}$ to reduce the related harmonic component of dc-link voltage. Namely, $i_{\mathrm{dc} 6}$ can be given by

$$
i_{\mathrm{dc} 6}=k \cdot i_{L 6}
$$

Where $k(0<k<1)$ is the corresponding gain coefficient, $i_{L 6}$ is the sixth harmonic component of $i_{L}$. Then, equation (6) can be rewritten as

$$
i_{\mathrm{dc}}=i_{\mathrm{dc} 0}+k \cdot i_{L 6}
$$

Further, substituting (8) into (5), the power of the equivalent load in Fig. 4 can be described as

$$
p_{e}=u_{\mathrm{dc}}\left(i_{\mathrm{dc} 0}+k \cdot i_{L 6}\right)
$$

Based on (9) and the dominant harmonic component of $u_{\mathrm{dc}}$, it can be deduced that sixth harmonic power and twelfth harmonic power are mainly contained in the HF components of the desired $p_{e}$. As the twelfth harmonic power is relatively small compared to the sixth harmonic power, it is reasonable to consider only the sixth harmonic power during the power control.

\subsection{Configuration of the proposed control scheme}

According to the PMSM mathematical model and the previous discussion, a control scheme for reducing dc-link voltage fluctuation is proposed in this paper, which takes into account two purposes: the PMSM speed regulation and the reduction of dc-link voltage harmonic component. The detailed configuration of the presented control scheme is shown in Fig. 5.

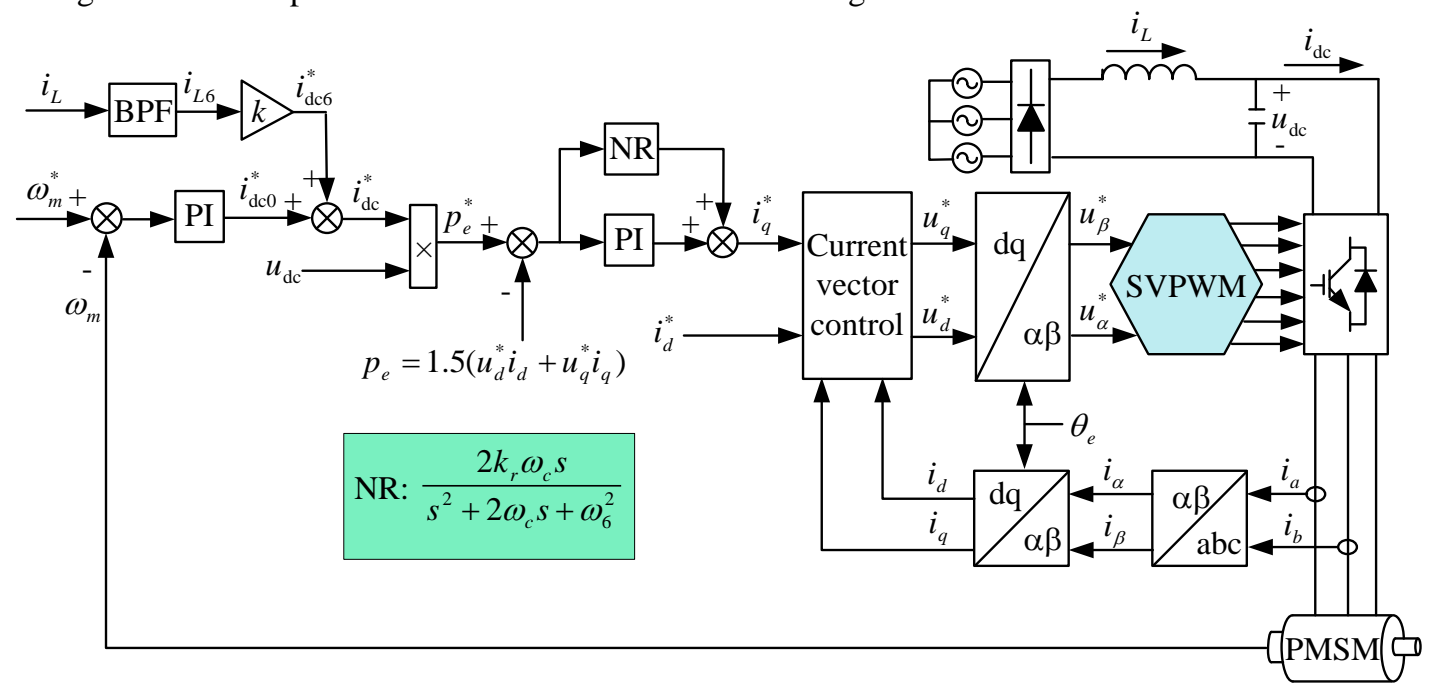

Figure 5. PMSM drive system with proposed control scheme.

In the proposed control scheme, due to the relatively high injected frequency of the motor electric power and the smoothing effect of mechanical inertia, the impact of the HF power injection on the motor speed $\omega_{m}$ can be neglected. This means that the HF components are almost not contained in the speed error, and hence, the output of speed regulator is designed as a DC form $i_{\mathrm{dc} 0}^{*}$, i.e., the command of $i_{\mathrm{dc} 0}$. Based on previous discussion, the reduction of dc-link voltage fluctuation can be achieved by shaping the sixth harmonic component of $i_{\mathrm{dc}}$ in phase with that of $i_{L}$. Consequently, the command of 
dc-link current sixth harmonic component $i_{\mathrm{dc} 6}^{*}$ is determined by a gain coefficient and $i_{L 6}$ generated from the band-pass filtering of $i_{L}$. Then, combining the sixth harmonic component command $i_{\mathrm{dc} 6}^{*}$ and the DC component command $i_{\mathrm{dc} 0}^{*}$, the dc-link current command that meet condition (8) is obtained. Further, assisted by the information of $u_{\mathrm{dc}}$, the power command $p_{\mathrm{e}}^{*}$ for the reduction of dc-link voltage fluctuation is obtained as well. After this, the power, which mainly contains DC component and sixth harmonic component, is regulated by the power controller. In detail, the DC power component is regulated according to the proportional plus integral (PI) controller, while the sixth harmonic power component is regulated according to the nonideal resonant (NR) controller [10] whose resonant frequency is designed as $\omega_{6}$ (i.e., six times the grid frequency). From such power regulation, the q-axis current command $i_{q}^{*}$ is generated. Then, the corresponding voltage commands are provided by the closed-loop control of current vectors. Finally, based on the coordinate transformation and space vector pulse with modulation (SVPWM), the desired duty cycles of the VSI are given.

In a word, with the aid of the closed-loop control of motor speed and reasonable HF power injection, the purposes of speed regulation as well as the reduction of dc-link harmonic voltage can be attained.

It can be observed from Fig. 5 that the presented method can be realized without the support of motor parameters, and thus, it is insensitive to motor parameters. Moreover, it is worth mentioning that the idea of reducing voltage fluctuation in the proposed control scheme can provide reference for other similar systems with reduced dc-link capacitor.

\section{Simulation results}

To verify the performance of the proposed control method for reducing dc-link voltage fluctuation, a simulation model of the PMSM drive system with reduced dc-link capacitor was built in MATLAB/Simulink, using the parameters listed in Table 1. Based on the established model, a series of simulation tests were performed.

Table 1. Simulation model parameters

\begin{tabular}{|c|c|}
\hline Parameter & Value \\
\hline Grid phase voltage/Frequency & $220 \mathrm{~V}(\mathrm{rms}) / 50 \mathrm{~Hz}$ \\
\hline DC side inductance $L$ & $0.35 \mathrm{mH}$ \\
\hline DC-link capacitance $C$ & $235 \mu \mathrm{F}$ \\
\hline Motor pole pairs & 4 \\
\hline Motor stator resistance $R$ & $0.6 \Omega$ \\
\hline Motor inductances $\left(L_{d} / L_{q}\right)$ & $5.1 \mathrm{mH} / 14.3 \mathrm{mH}$ \\
\hline Motor permanent magnet flux linkage & $0.17 \mathrm{~V} . \mathrm{s}$ \\
\hline
\end{tabular}

Fig. 6 shows the motor speed response with 8 N.m load torque when the speed command 2000 $\mathrm{r} / \mathrm{min}$ is given, and the corresponding motor electric power is shown in Fig. 7. The speed response indicates that the proposed method has well capability of regulating motor speed. Moreover, according to the steady-state motor electric power and motor speed, it can be known that the HF injection for the motor electric power does not cause obvious HF speed ripples. Therefore, the PMSM speed control performance is guaranteed, although the system dc-link capacitor is reduced. 


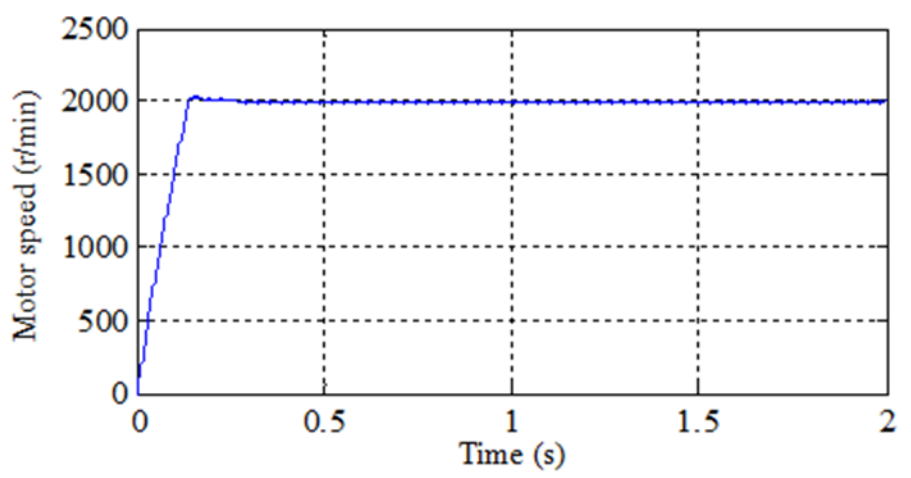

Figure 6. Motor speed waveform for the proposed method with 8 N.m load torque.

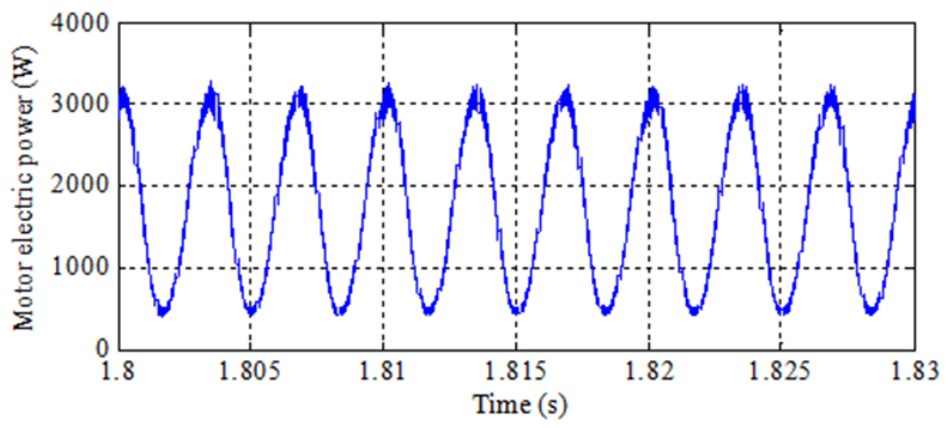

Figure 7. PMSM electric power for the proposed method at $2000 \mathrm{r} / \mathrm{min}$ with 8 N.m load torque.

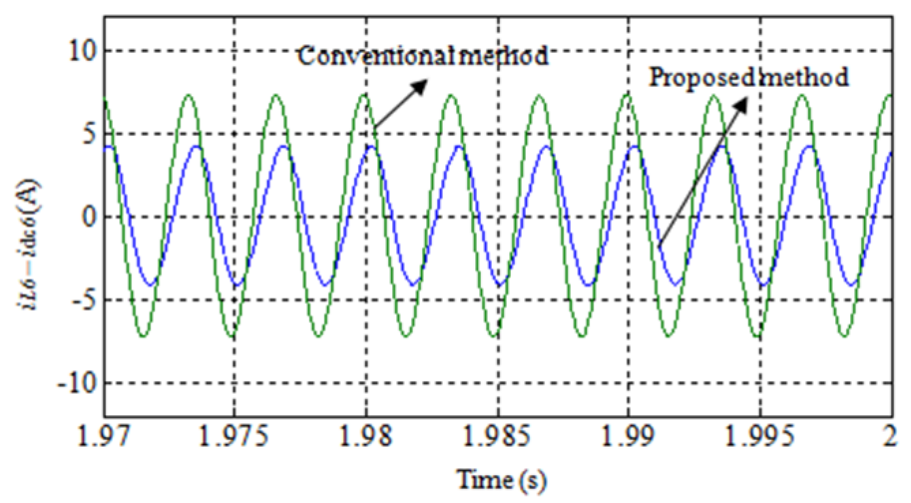

Figure 8. Sixth harmonic current difference comparison under $p_{e}=2.0 \mathrm{~kW}$.

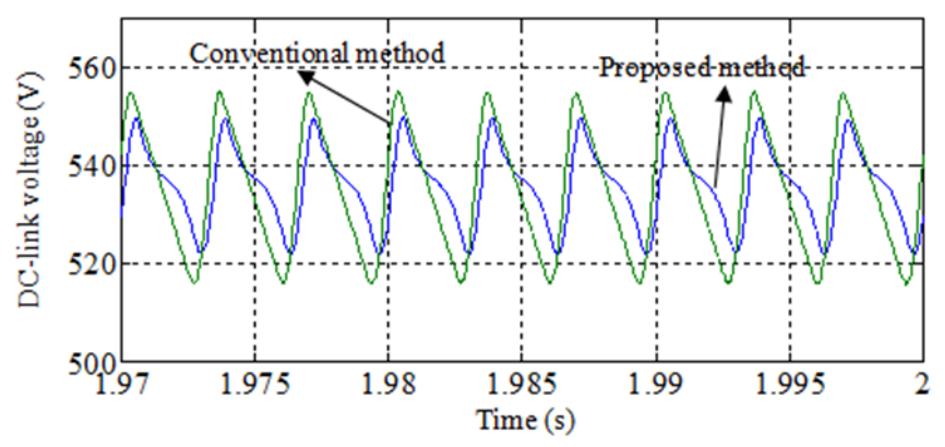

Figure 9. DC-link voltage comparison under $p_{e}=2.0 \mathrm{~kW}$. 
In order to demonstrate that the proposed scheme can reduce the voltage fluctuation of the motor drive system with reduced dc-link capacitor, the conventional field-oriented control method (hereafter referred to as conventional method) and the proposed method were performed under the same condition (i.e., $p_{e}=2.0 \mathrm{~kW}$ ), respectively, and the results were shown in Fig. 8 and Fig. 9. It can be seen from these curves that, under the control of conventional method, the difference between $i_{L 6}$ and $i_{\mathrm{dc} 6}$ is $7.2 \mathrm{~A}$ (amplitude), and the dc-link voltage ripple is $39.3 \mathrm{~V}$; under the control of proposed method, the difference between $i_{L 6}$ and $i_{\mathrm{dc} G}$ is reduced to $4.2 \mathrm{~A}$ (amplitude), and the dc-link voltage ripple is reduced to $27.9 \mathrm{~V}$, accordingly. This confirms that the reasonable shaping of dc-link current by power regulation can reduce the dc-link voltage fluctuation. In other words, the method in this paper is feasible.

In addition, to further demonstrate the performance of the proposed method, the dc-link voltage ripples of the conventional PMSM control method and the proposed method are summarized in Fig. 10. From this comparison, it can be seen that the voltage ripple of the proposed method is lower than that of the conventional method regardless the DC component of motor electric power. This also indicates that the system with reduced dc-link capacitor is stable.

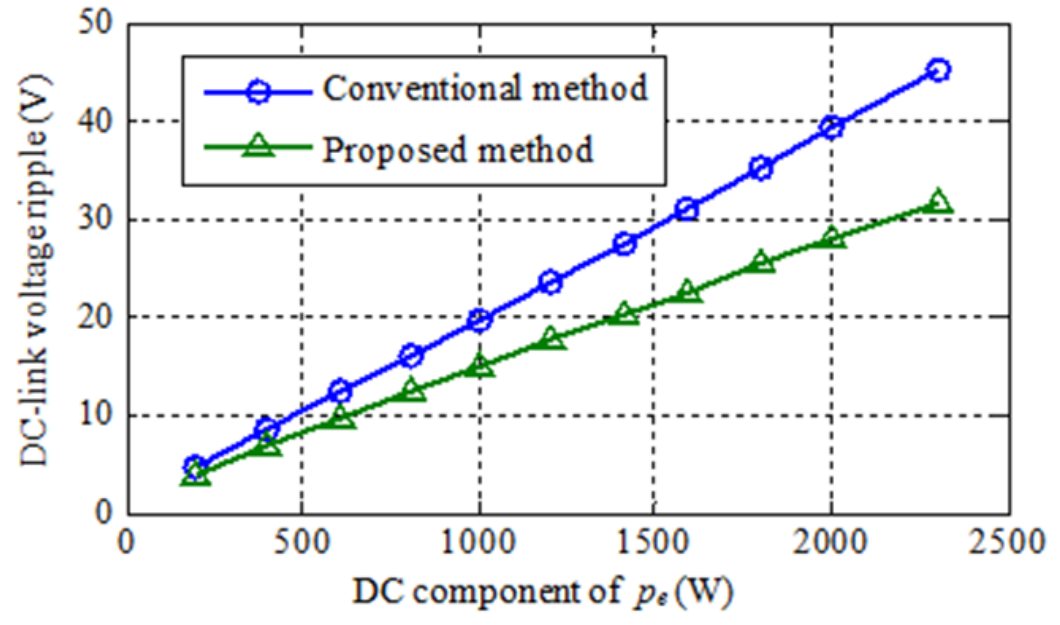

Figure 10. DC-link voltage ripples comparison between conventional method and the proposed method.

In short, these simulation tests confirm that the proposed control scheme can not only regulate the motor speed but also improve the ripple characteristic of the dc-link voltage.

\section{Conclusions}

In this paper, the dc-link voltage fluctuation problem in a PMSM drive system with reduced dc-link capacitor has been investigated, and the VSI power condition of reducing this voltage fluctuation has been analyzed accordingly. Based on the deduced power condition, a novel control method to reduce the dc-link voltage ripple has been proposed. The proposed method is independent of motor parameters. Simulation results confirm that the presented approach can meet the requirements of both the PMSM speed regulation and the reduction of dc-link voltage fluctuation.

\section{Acknowledgments}

This work was supported by the Shenzhen Science and Technology Plan Project under Grant JCYJ20150403161923537. 


\section{References}

1. P. Pillay and R. Krishnan, "Modeling, simulation, and analysis of permanent-magnet motor drives, part I: the permanent-magnet synchronous motor drive”, IEEE Trans. Ind. Appl., 25, 2, pp. 265273, (1989)

2. X. Zhang and Z. Li, "Sliding-mode observer-based mechanical parameter estimation for permanent magnet synchronous motor”, IEEE Trans. Power Electron., 31, 8, pp. 5732-5745, (2016)

3. K. Inazuma, K. Ohishi, and H. Haga, "High-power-factor control for inverter output power of IPM motor driven by inverter system without electrolytic capacitor”, in Proc. IEEE International Symposium on Industrial Electronics, pp. 619-624, (2011)

4. Y. Son and J.-I. Ha, "Direct power control of a three-phase inverter for grid input current shaping of a single-phase diode rectifier with a small DC-link capacitor”, IEEE Trans. Power Electron., 30, 7, pp. 3794-3803, (2015)

5. H. Yoo and S.-K. Sul, "A new circuit design and control to reduce input harmonic current for a three-phase AC machine drive system having a very small DC-link capacitor ", in Proc. $25^{\text {th }}$ Annual IEEE Applied Power Electronics Conference and Exposition, pp. 611-618, (2010)

6. W.-J. Lee and S.-K. Sul, "DC-link voltage stabilization for reduced DC-link capacitor inverter”, IEEE Trans. Ind. Appl., 50, 1, pp. 404-414, (2014)

7. B.-G. Gu and K. Nam, "A DC-link capacitor minimization method through direct capacitor current control”, IEEE Trans. Ind. Appl., 42, 2, pp. 573-581, (2006)

8. J. Kim, I. Jeong, K. Lee, and K. Nam, "Fluctuating current control method for a PMSM along constant torque contours”, IEEE Trans. Power Electron., 29, 11, pp. 6064-6073, (2014)

9. P. Magne, D. Marx, B. Nahid-Mobarakeh, and S. Pierfederici, "Large-signal stabilization of a DC-link supplying a constant power load using a virtual capacitor: impact on the domain of attraction”, IEEE Trans. Ind. Appl., 48, 3, pp. 878-887, (2012)

10. H. Komurcugil, N. Altin, S. Ozdemir, and I. Sefa, "Lyapunov-function and proportional-resonantbased control strategy for single-phase grid-connected VSI with LCL filter”, IEEE Trans. Ind. Electron., 63, 5, pp. 2838-2849, (2016) 